\title{
Therapeutic and Protective Effects of Pharmacological Agents on Femoral Head Osteonecrosis
}

\section{Z. ZHAO*}

Department of Joint Surgery, Xintai City People's Hospital, No. 1329, Xinfu Road, Xintai City, Shandong Province 271200, China

\section{Zhao: Effects of Pharmacological Agents on FHO}

\begin{abstract}
A variety of pharmacological agents are available for the treatment of osteonecrosis of the femoral head. Nonetheless, several controversies are associated with their clinical use. Various electronic databases such as Medline (19992019) and EMBASE (1999-2019) were searched for an extensive literature review in English using various keywords including, femoral head osteonecrosis and pharmacological treatments together. The retrieved publications were carefully reviewed to find out the efficacy and safety of the pharmacological agents used for the treatment of femoral head osteonecrosis. The presently used pharmacological treatments in clinical practice for femoral head osteonecrosis included iloprost, enoxaparin, bisphosphonates, and statins. Nonetheless, studies have reported poor efficacy and several undesired effects of these agents. None of the high-level studies recommended the therapeutic and protective use of pharmacological agents in the treatment of osteonecrosis of the femoral head.
\end{abstract}

Key words: Osteonecrosis, pharmacological agents, efficacy, safety

Osteonecrosis of the femoral head is a pathological condition that is triggered by the reduction of blood flow to the joints area, which results in the collapse of the necrotic portion leading to developing osteoarthritis of the hip joint. This is a more common finding in young people leading to total hip arthroplasty (THA) ${ }^{[1,2]}$. Its incidence in the general population is increasing due to the heavy use of steroids as a combination therapy for the treatment of malignant neoplasms and organ transplant recovery ${ }^{[3-5]}$. According to an estimate, 10000 to 20000 newly admitted patients are affected by this disease ${ }^{[6]}$. After surveying the whole of South Korea, it is estimated that the occurrence of the disease was more than $10000^{[7]}$. Over time, the disease would become a socioeconomic problem. There are many published reports during the period of January 1999 to December 2018, which explored the effects of the treatment related to osteonecrosis. Two meta-analyses 
carried out on the treatment of osteonecrosis with bisphosphonates clearly pointed out that the usefuness is limited due to the low efficacy of bisphosphonates ${ }^{[8,9]}$. At III/IV level studies, a small number of patients were examined. Meta-analysis was not applied for treatment purpose at the I/II level study due to insufficient data. Hence publications from the past $20 \mathrm{y}$ were retrieved to understand the latest mechanisms and pharmacological treatments that were more effective in treating femoral head osteonecrosis (FHO).

Several genetic and risk factors are responsible for the development of FHO. Ischemia is the main cause of the disease. The pathophysiology of this disease involved marrow necrosis and osteocyte death; healing of necrotic part of bone and breakage of necrotic bone and development of osteoarthritis of the hip ${ }^{[10]}$ (fig. 1).

Steroids and alcohols exert many effects on the femoral head such as increased size of marrow fat cells, intraosseous hypertension, venous sinusoidal obstructive syndrome and disseminated intravascular coagulation. The obstruction of blood flow results in the development of ischemia and marrow necrosis. The pathophysiology of FHO is thrombophilia and impairment of generating new vessels (angiogenesis). In the absence of these factors, the pathological conditions reversed and did not disseminate, while in the presence of the above factors, the prolonged ischemic and necrotic conditions led to the formation of sequestrum. When sequestrum was ostensible viz. greater than $1 \mathrm{~cm}$, the bone did not undergo creeping substitution, Subsequently, foreign or macrophages response started around sequestrum in which histiocytes and giant cells started aggregating and formed a fibrous capsule. Afterward, in MRI it looked just like band lesions. In the sequestrum, fatty acid released from dead marrow formed soap after saponification. After all the process, dead bone and saponified marrow did not gain the previous strength and at the sub-chondral portion, a fatigue fracture develops which resulted in osteoarthritis of the hip. The further progress of this ischemic disease to osteonecrosis depended on the restoring capability from fibrinolysis and angiogenesis which in result help osteocytes to work properly.

There are several methods to treat FHO. The nonsurgical methods adopt at the pre-collapsed stage is a weight-bearing restriction, using a cane, crutches or a walker and alteration in the activity. At an early stage, these methods can be helpful but do not prevent the disease from developing ${ }^{[5,11-20]}$. Preventing the patient from lifting weight can only help if the patient has a limited disease and cannot undergo surgery due to any reason ${ }^{[21,22]}$. The other supplemental treatments include the use of pharmacological agents such as anticoagulants, traditional Chinese medicines, bisphosphonate, and other non-invasive tools that are used for measuring metabolism and biophysical modalities such as electromagnetic stimulation and hyperbaric oxygen ${ }^{[7,23-32]}$. These drugs use specific pathways to combat the disease. If the bone condition is good, it is necessary to arrest the process of osteonecrosis with pharmacological approaches ${ }^{[33]}$. These methodologies include physiotherapy of a specific joint. According to a report, it is evident that the old methodologies are not beneficial for the treatment of $\mathrm{FHO}^{[3]}$. Various pathological mechanisms have been adopted and practiced to cure this disease.

Enoxaparin was used for the treatment of earlystage osteonecrosis as reported by Glueck et al. ${ }^{[11]}$. These authors documented that enoxaparin improved fibrinolysis of intravascular thrombi, which could results in improvement of blood flow, reverse the ischemic condition and initiated the healing process. Patients treated with enoxaparin $60 \mathrm{mg}$ for 3 mo were divided into 2 groups one with 16 patients ( 25 hips)

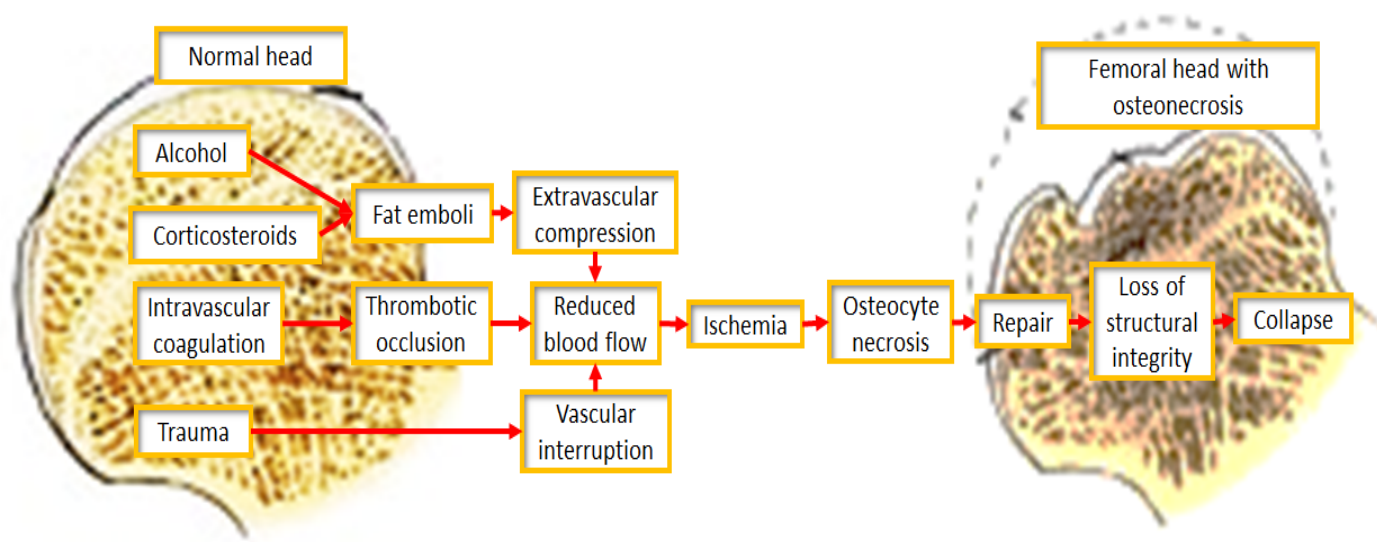

Fig. 1: Schematic representation of processes involved in the osteonecrosis of femoral head. 
with thrombophilic-hypofibrinolytic-induced primary osteonecrosis and the other one with 12 patients (15 hips) with alcohol or steroid-induced secondary osteonecrosis. It was reported that $12 \%$ of patients with primary osteonecrosis had a femoral head collapse; still, enoxaparin did not inhibit further progression of the disease. Moreover, after the trial, it was also concluded that out of 28 , there were 6 patients with familial thrombophilia, in whom the disease did not progress for 4 to $16 \mathrm{y}$ after long-term treatment with oral anticoagulants. Similarly Chotanaphuti et al. documented 36 patients with osteonecrosis of the femoral head at an early stage ${ }^{[13]}$. The total number of patients were divided into two groups; both with eighteen patients each (26 hips). One of the groups received a daily dosage of 6000 units of enoxaparin for $12 \mathrm{w}$, while the other one did not take any medicines. In the study group, $57.7 \%$ of patients remained in the pre-collapse stage after 2 y of minimum follow-up. While on the other hand, in the control group $21.7 \%$ of patients remained in the pre-collapsed stage and the p-value found to be 0.042 . However, it can be seen that there were no serious harmful effects along with bleeding seen in this study. It should also be made clear from a study that the balance between bleeding risk and the benefit of osteonecrosis should remain significant.

Glucocorticoids increase the risk of osteonecrosis by increasing fat content in the bone marrow. Cardiovascular risk associated with lipid-lowering agents was decreased by the use of Statins. It was also confirmed from studies that statins showed a protective effect against osteonecrosis in steroid users ${ }^{[14,15]}$. Pritchett reported that there were 284 patients treated with high dose steroids and followed for a period of $5 \mathrm{y}^{[14]}$. The results indicated that during the time period of $5 \mathrm{y}$, only $1 \%$ of patients developed osteonecrosis. The trend of generating osteonecrosis was a lot less than the previous incidence, which showed a value of 3 to $20 \%$ in patients receiving high-dose steroids. Thus statins were reported by Ajmal et al. to find out the decreasing trend of glucocorticoid-induced osteonecrosis and collected the database of 2,881 renal transplantation patients, in which only $15(4.4 \%)$ developed osteonecrosis ${ }^{[16]}$. The other 180 (7\%) who were not using glucocorticoids developed the disease. The percentage of patients who did not show osteonecrosis was similar in both patients using statins and patients who did not use statins.

There are only a few level 3 or level 4 human studies that provided evidence for the protective effect of statins in osteonecrosis and there were no randomized trials based on level 1 or level 2 studies. The long term use of statins might produce some kind of risk such as diabetes, liver damage and induce cognitive disorders ${ }^{[17]}$. Thus for the protective use of statins, it must undergo randomized controlled clinical trials with risk-benefit assessment against osteonecrosis.

Bisphosphonates are a class of drugs, which prevent loss of bone density and stop osteoclast activity by promoting the healing process. Previous studies documented that in osteonecrosis patients alendronate has a significant role in preventing femoral head collapse. Later in 2005, Lai et al. reported that alendronate can prevent femoral head collapse ${ }^{[18]}$. They started the experiment with 40 patients (54 hips) with early-stage FHO and incorporated alendronate in one group and took others as a control group. The treatment period duration was of $25 \mathrm{w}$ and the treatment protocol was a dose of $70 \mathrm{mg}$ of oral alendronate every week. After $2 \mathrm{y}$ of follow up treatment with alendronate, 2 out of 29 femoral heads have collapsed. While on the other hand, in the control group 19 out of 25 femoral heads collapsed. Out of all the above data, one hip in the alendronate group under-went the THA while in the control group 16 hips underwent this surgery ${ }^{[18]}$.

Agarwala et al. reported a trial on 60 patients (100 osteonecrosis hips) by treating them with alendronate with a dose of $10 \mathrm{mg} / \mathrm{d}$ in combination with 500 to $1,000 \mathrm{mg}$ of daily calcium and vitamin D supplements ${ }^{[19]}$. It was evident from increased walking time and from pain relief that this combination has a significant effect. The hip movement gradually improved during $1 \mathrm{y}$ of treatment and then the process slowed down. After radiographic investigation it was observed that the disease advanced to grade 1 and then stabilized. From MRI it was noticed that marrow edema was minimal in most cases. Out of them, only 6 patients needed to go for surgery. Nishii et al. also documented the effect of alendronate on femoral head necrosis ${ }^{[20]}$. The results reported were quite similar as the earlier studies with 13 hips out of 60 hips collapsed in the control group and 1 out of 20 hips collapsed in the alendronate-treated group.

However, it was found out later that alendronate does not play any role in reducing disease process or prevent surgery such as THA. The prospective, randomized and double-blind placebo-controlled study reported by Chen et al..$^{[21]}$, involved 64 patients and 2 y to complete the study. Out of the 64 patients with osteonecrosis 
enrolled, 52 patients were assessed for final analysis. It was found that 4 out of 32 hips in the alendronate group were undergone THA while 5 out of 33 hips in the placebo control group underwent THA. This difference in disease progression was considered as minimal showed 36 scores between these 2 groups.

Another 2 y study associated with early-stage osteonecrosis having a necrotic area $(>30 \%)$ was reported by Lee et al. ${ }^{[22]}$. The randomized, open-label trial started with 110 patients to find out the effect of zoledronate in the prevention of collapse and reduction in the incidence of THA. Out of 110, 55 patients were treated with zoledronate with dose of $5 \mathrm{mg}$ intravenously per year for $2 \mathrm{y}$ while the remaining 55 patients in the control group were not treated with zoledronate. After a 2 y follow-up it was observed that 29 femoral heads in the zoledronate group were collapsed while 22 femoral heads in the control group were collapsed. Moreover, 19 hips in the zoledronate-treated group underwent THA while 20 hips in the control group underwent THA. Thus from this study, it was clear that zoledronate did not prevent femoral head collapse nor reduce the necessity of surgery such as THA.

The bisphosphonate potency was determined through 2 meta-analyses ${ }^{[8,9]}$. Firstly, Yuan et al. identified 5 eligible trials in one meta-analysis ${ }^{[8]}$. The trials comprised 329 subjects in which with 920.9 patient-y of follow-up, it was found that bisphosphonates neither prevent femoral head collapse nor prevent the necessity of THA. The Haris hip score was not improved by bisphosphonates. Li et al. estimated the efficacy of bisphosphonates in a study on 16 animal and 7 human trials ${ }^{[9]}$. The results documented from animal studies showed that bisphosphonates improved bone remodelling, although it was established previously that bisphosphonates did not play a role in hip pain management, femoral head collapse, necessity for THA or Harris hip score. The adverse effects of bisphosphonates found were, esophageal or gastric irritation while osteonecrosis of the jaw and insufficiency fractures of the femur were also reported ${ }^{[23]}$. Hence bisphosphonates are not usually prescribed in patients with FHO because of their unclear protective effect and associated adverse effects.

Iloprost is a synthetic analog of prostacyclin PGI2. It showed activity as a dilator of systemic and pulmonary vascular beds. There has been clinical evidence for its use in the treatment of osteonecrosis. Jager et al. reported in a trial comprised of 95 patients ( 186 bones) with the disease of bone marrow edema or osteonecrosis ${ }^{[24]}$, after a 1 y and 8 mo of follow-up treatment it was found that the pain was relieved and functional mobility increased.

The iloprost-treated joints were further documented by Claben et al. ${ }^{[25]}$. The trial reported treatment of 108 patients in which 136 osteonecrosis joints were considered for treatment. The follow-up time for these patients was set at $50 \mathrm{mo}$, after treatment with iloprost and $74.8 \%$ of patients showed decreased visual analog scale pain scores. Though $20 \%$ of them underwent total joint arthroplasty as they were associated with the Association Research Circulation Osseous (ARCO) stage $2,71 \%$ with ARCO stage 3 and $100 \%$ with ARCO stage 4 . The heterogeneous patient population along with the un-estimated size of the necrotic portion was considered as the major limitation, which proceeded it to collapse.

Iloprost has many adverse effects most common of those were, hypotension, flushing, cough, headache, flu syndrome, nausea, vomiting, hypotension, insomnia and palpitation. While congestive heart failure, supraventricular tachycardia, and renal failure are serious adverse effects associated with iloprost ${ }^{[26]}$. Although Jager et al..$^{[24]}$ and Claben et al. ${ }^{[25]}$ collectively found out that iloprost has a significant effect on bone marrow edema syndrome and early-stage osteonecrosis. As described previously that bone marrow edema syndrome and ischemic lesion did not definitely develop osteonecrosis ${ }^{[10]}$. Still, additional research is required on the safety and effect of iloprost.

Aspirin is also known as acetylsalicylic acid which binds to and acetylates serine in cyclooxygenases and thus inhibited the synthesis of prostaglandins. It works by inhibiting aggregation of platelets and prevent arterial and venous thrombosis, reduce pain and inflammation. Albers et al. in a trial with 10 patients (12 hips) suffering from pre-collapsed $\mathrm{FHO}{ }^{[27]}$ gave a daily dose of $81 \mathrm{mg}$ of aspirin and fund that femoral head collapse was seen in only 1 patient ( $8 \%$ ) after a 3.7 y of follow-up treatment. Though this study involved only 10 patients in which 9 of the patients had type B or C1 lesions according to the Japanese Investigation Committee ${ }^{[28]}$, these lesions are further at risk of collapse.

After each trial the efficacy of pharmacological treatment should be monitored cautiously. For this purpose, the pain intensity in the early stage and the zone of the necrotic portion should be evaluated. When osteonecrosis starts after an acute ischemic attack marrow edema started to develop around the necrotic portion of the femoral head. Additionally, the patients 
suffer from severe pain even before collapse occurs. As the edema resolves, the pain reduces, with or without any treatment. The sudden resting pain might be caused by the use of medications. The collapse of the femoral head is not seen in small lesions without any medical or surgical intervention. With time many new medical interventions have been introduced. Though these methods really prevent the disease from progression or reverses to its normal stage is still unclear. Considering a lot of risks there is no clear evidence for pharmacological prevention or treatment of osteonecrosis recommended at this moment.

\section{Conflict of interest:}

No conflict of interest between any of the authors.

\section{REFERENCES}

1. Schneider W, Knahr K. Total hip replacement in younger patients: survival rate after avascular necrosis of the femoral head. Acta Orthop Scand 2004;75:142-6.

2. Hungerford DS. Treatment of osteonecrosis of the femoral head: everything's new. J Arthroplasty 2007;22:91-4.

3. Shibatani M, Fujioka M, Arai Y, Takahashi K, Ueshima K, Okamoto $\mathrm{M}$, et al. Degree of corticosteroid treatment within the first 2 months of renal transplantation has a strong influence on the incidence of osteonecrosis of the femoral head. Acta Orthop 2008;79:631-6.

4. Arlet J. Nontraumatic avascular necrosis of the femoral head. Past, present, and future. Clin Orthop Relat Res 1992;277:1221.

5. Shigemura T, Nakamura J, Kishida S, Harada Y, Ohtori S, Kamikawa $\mathrm{K}$, et al. Incidence of osteonecrosis associated with corticosteroid therapy among different underlying diseases: prospective MRI study. Rheumatology (Oxford) 2011;50:2023-8.

6. Hungerford DS. Osteonecrosis: avoiding total hip arthroplasty. J Arthroplasty 2002;17:121-4.

7. Kang JS, Park S, Song JH, Jung YY, Cho MR, Rhyu KH. Prevalence of osteonecrosis of the femoral head: a nationwide epidemiologic analysis in Korea. J Arthroplasty 2009;24:117883.

8. Yuan HF, Guo CA, Yan ZQ. The use of bisphosphonate in the treatment of osteonecrosis of the femoral head: a meta-analysis of randomized control trials. Osteoporos Int 2016;27:295-9.

9. Li D, Yang Z, Wei Z, Kang P. Efficacy of bisphosphonates in the treatment of femoral head osteonecrosis: A PRISMA compliant meta-analysis of animal studies and clinical trials. Sci Rep 2018;8:1450-7.

10. Koo KH, Jeong ST, Jones JP Jr. Borderline necrosis of the femoral head. Clin Orthop Relat Res 1999;358:158-65.

11. Glueck CJ, Freiberg RA, Sieve L, Wang P. Enoxaparin prevents the progression of stages I and II osteonecrosis of the hip. Clin Orthop Relat Res 2005;435:164-70.

12. Glueck CJ, Freiberg RA, Wissman R, Wang P. Long term anticoagulation (4-16 y) stops progression of idiopathic hip osteonecrosis associated with familial thrombophilia. Adv Orthop 2015;2015:138382.

13. Chotanaphuti T, Thongprasert S, Laoruengthana A. Low molecular weight heparin prevents the progression of precollapse osteonecrosis of the hip. J Med Assoc Thai 2013;96:1326-30.

14. Pritchett JW. Statin therapy decreases the risk of osteonecrosis in patients receiving steroids. Clin Orthop Relat Res 2001;386:173-8.

15. Pengde K, Fuxing P, Bin S, Jing Y, Jingqiu C. Lovastatin inhibits adipogenesis and prevents osteonecrosis in steroidtreated rabbits. Joint Bone Spine 2008;75:696-701.

16. Ajmal M, Matas AJ, Kuskowski M, Cheng EY. Does statin usage reduce the risk of corticosteroid-related osteonecrosis in renal transplant population? Orthop Clin North Am 2009;40:235-9.

17. U.S. Food and Drug Administration. FDA Drug Safety Communication: Important safety label changes to cholesterollowering statin drugs. 2012.

18. Lai KA, Shen WJ, Yang CY, Shao CJ, Hsu JT, Lin RM. The use of alendronate to prevent early collapse of the femoral head in patients with nontraumatic osteonecrosis. A randomized clinical study. J Bone Joint Surg Am 2005;87:2155-9.

19. Agarwala S, Shetty V, Karumuri LK, Vijayvargiya M. Patellar resurfacing versus nonresurfacing with patellaplasty in total knee arthroplasty. Indian J Orthop 2018;52:393-8.

20. Nishii T, Sugano N, Miki H, Hashimoto J, Yoshikawa H. Does alendronate prevent collapse in osteonecrosis of the femoral head? Clin Orthop Relat Res 2006;443:273-9.

21. Chen $\mathrm{CH}$, Chang JK, Lai KA, Hou SM, Chang CH, Wang GJ. Alendronate in the prevention of collapse of the femoral head in nontraumatic osteonecrosis: a two-year multicenter, prospective, randomized, double-blind, placebo-controlled study. Arthritis Rheum 2012;64:1572-8.

22. Lee YK, Ha YC, Cho YJ, Suh KT, Kim SY, Won YY, et al. Does zoledronate prevent femoral head collapse from osteonecrosis? A prospective, randomized, open-label, multicenter study. J Bone Joint Surg Am 2015;97:1142-8.

23. Pazianas M, Abrahamsen B. Safety of bisphosphonates. Bone 2011;49:103-10.

24. Jager M, Zilkens C, Bittersohl B, Matheney T, Kozina G, Blondin D, et al. Efficiency of iloprost treatment for osseous malperfusion. Int Orthop 2011;35:761-5.

25. Claben T, Becker A, Landgraeber S, Haversath M, Li X, Zilkens C, et al. Long-term clinical results after iloprost treatment for bone marrow edema and avascular necrosis. Orthop Rev 2016;8:6150-6.

26. Pountos I, Giannoudis PV. The role of Iloprost on bone edema and osteonecrosis: safety and clinical results. Expert Opin Drug Saf 2018;17:225-33.

27. Albers A, Carli A, Routy B, Harvey EJ, Séguin C. Treatment with acetylsalicylic acid prevents short to mid-term radiographic progression of nontraumatic osteonecrosis of the femoral head: a pilot study. Can J Surg 2015;58:198-205.

28. Sugano N, Atsumi T, Ohzono K, Kubo T, Hotokebuchi T, Takaoka K. The 2001 revised criteria for diagnosis, classification, and staging of idiopathic osteonecrosis of the femoral head. J Orthop Sci 2002;7:601-5.

29. Koo KH, Ahn IO, Kim R, Song HR, Jeong ST, Na JB, et al. Bone marrow edema and associated pain in early stage osteonecrosis of the femoral head: prospective study with serial MR images. Radiology 1999;213:715-22.

30. Ha YC, Jung WH, Kim JR, Seong NH, Kim SY, Koo KH. Prediction of collapse in femoral head osteonecrosis: a 
modified Kerboul method with use of magnetic resonance images. J Bone Joint Surg Am 2006;88:35-40.

31. Steinberg ME, Hayken GD, Steinberg DR. A quantitative system for staging avascular necrosis. J Bone Joint Surg $\mathrm{Br}$ 1995; 77:34-41.

32. Chen $\mathrm{CH}$, Chang JK, Lai KA, Hou SM, Chang $\mathrm{CH}$, Wang GJ. Alendronate in the prevention of collapse of the femoral head in nontraumatic osteonecrosis: a two-year multicenter, prospective, randomized, double-blind, placebo-controlled study. Arthritis Rheum 2012;64:1572-8.

33. Wang C, Peng J, Lu S. Summary of the various treatments for osteonecrosis of the femoral head by mechanism: a review. Exp Ther Med 2014;8:700-6.

This is an open access article distributed under the terms of the Creative Commons Attribution-NonCommercial-ShareAlike 3.0 License, which allows others to remix, tweak, and build upon the work non-commercially, as long as the author is credited and the new creations are licensed under the identical terms

This article was originally published in a special issue, "Biomedical research applications in Pharmaceutical Sciences"

Indian J Pharm Sci 2020:82(2)spl issue3;16-21 